\title{
Analysis and Result Confirmation of Cohesive Subgroup Structure among Classmates
}

\author{
W.Y. ZHU \\ Jiangsu Tianyi High School, Wuxi, Jiangsu, China \\ J.H. GAO \\ Shanghai Center for Bioinformation Technology, Shanghai, China
}

\begin{abstract}
This article utilizes social network method to analyze the relationships among middle school classmates of several classes in Wuxi, Jiangsu. Software including UCINET and NetDraw generates designated number of cohesive subgroups within the classes and the process of subgroup division and development is studied. With Girvan-Newman Algorithm, q-value of each subgroup is calculated and confirmed by each class teacher's feedback on the characteristics of each subgroup. Parameters including Degree, EcCentricity, Closeness and Betweenness of key individuals calculated by software Cytoscape prove to largely correspond with the description of the class teachers.
\end{abstract}

KEYWORD: Social network analysis; Cohesive subgroup structure; Classes; Classmates; Information distribution; UCINET; NetDraw

\section{RESEARCH PURPOSE}

This article refers to the research methods in document[1][2], analyzes the relationships among classmates with social network method, studies the cohesive subgroups within classes and looks for patterns which are applied in solving practical problems.

\section{DATA COLLECTION}

Questionnaires were utilized in the collection of data. Each student was required to fill in their name, gender, three students they like and three students they dislike. Researchers promised the confidentiality beforehand in order to ensure the accuracy of the resulting information.

Sort out the collected data. For students along the column, mark in the corresponding grid along the row the classmates they like with + sign, those they dislike with - sign, and the space of their own number with to indicate "not applicable". The relationships among the 41 students in Class A are presented in Table 1 .
Table 1. Relationships among students in class A.

\begin{tabular}{|c|c|c|c|c|c|c|}
\hline Gender & Number & 1 & 2 & 3 & $\ldots$ & 41 \\
\hline Male & 1 & 口 & & & & \\
\hline Female & 2 & - & घ & & & + \\
\hline \multirow[t]{2}{*}{ Male } & 3 & & & a & & \\
\hline & $\ldots$ & & & & घ & \\
\hline \multirow[t]{3}{*}{ Female } & 41 & - & + & & & घ \\
\hline & $\operatorname{SUM}(+)$ & 1 & 6 & 3 & & 3 \\
\hline & SUM(-) & 14 & 2 & 1 & & 1 \\
\hline
\end{tabular}

\section{STRUCTURAL ANALYSIS OF "LIKE" RELATIONSHIP NETWORK AMONG CLASSMATES}

Table 1 can be used to generate a matrix of "like" relationships among classmates. The entries in the matrix include only 1 and 0 . If the student in row $\mathrm{i}$ likes the student in column $\mathrm{j},(\mathrm{i}, \mathrm{j})=1$. Otherwise, $(\mathrm{i}$, $\mathrm{j})=0$. Import the matrix file into UCINET [3], and a corresponding \#\#h format file will be generated automatically. Import \#\#h file into NetDraw [4] and Figure 1 represents the "like" relationship network among students of Class A. 


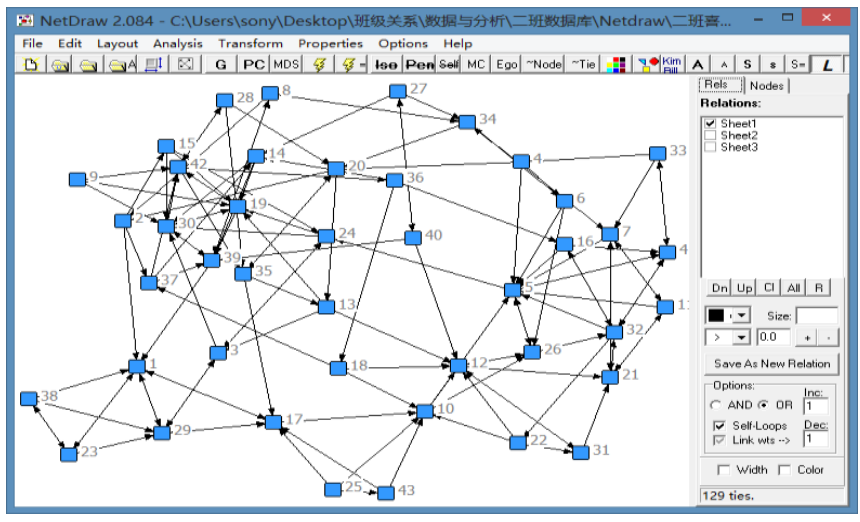

Figure 1. "Like" relationship network of class A.

Generate cohesive subgroups within the "like" relationship network with "Factions", and the result of five factions is presented in Figure 2. It can be observed that vertices gather together according to subgroups and each subgroup is represented by a distinct color.

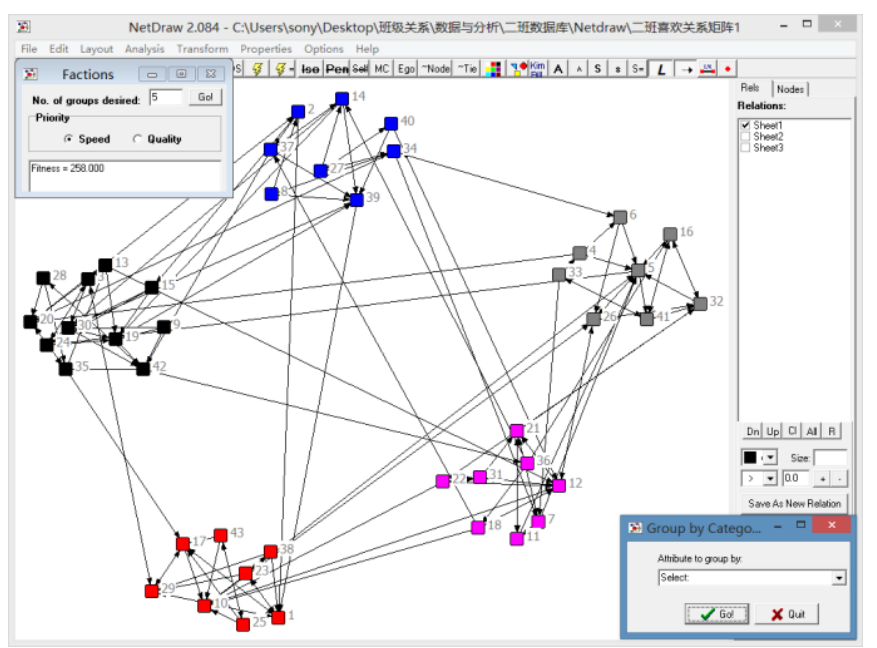

Figure 2. Cohesive subgroup structure of class A with faction $=5$.

\section{ANALYSIS AND RESULT CONFIRMATION OF COHESIVE SUBGROUP DIVISION}

With regard to the "like" relationship network of Class A and B, analyze how the cohesive subgroups divide from large to small and compare the theoretical result with the actual situation.

\subsection{Class A}

While the class is divided into two major subgroups (Figure 3-(a)), gender is the main determinant factor of division. The subgroups targeted by the research is generally consisted of one single gender - female. When classmates divide further into smaller subgroups, girls number $4,5,6,16,26,32,33,41$ are always in the same subgroup. Even as the total number of subgroups increase, the relationship among these students is not influenced (Figure 3-(b), $3-(c))$. According to the feedback from class teachers, students number 4,5 , and 6 are the best friends. Therefore, the female subgroup based on the strong friendship is quite stable.

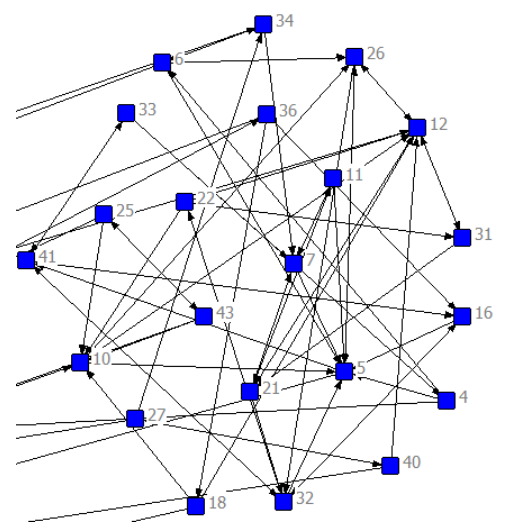

(a)Two Factions

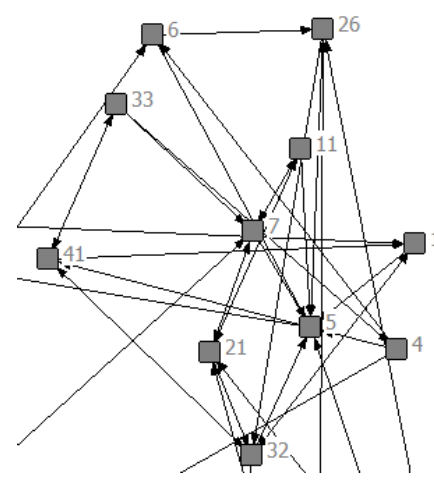

(b) Four Factions

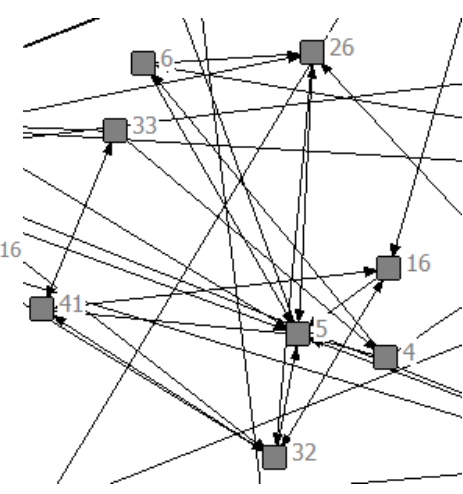

(c) Seven Factions
Figure 3. Division process of cohesive subgroups in class A.

Calculation of core figures' network parameters by Hubba[6] plug-in in Cytoscape[5]:

Table 2. Hubba calculation of class A core figures' network parameters.

\begin{tabular}{|c|c|c|c|}
\hline Node Name & Degree & Closeness & Betweenness \\
\hline 4 & 4 & 18.17 & 29.19 \\
\hline 33 & 4 & 15.47 & 5.31 \\
\hline 6 & 5 & 17.98 & 17.13 \\
\hline 16 & 6 & 17.90 & 25.79 \\
\hline 26 & 7 & 19.42 & 31.95 \\
\hline 32 & 7 & 17.90 & 14.92 \\
\hline 41 & 7 & 16.90 & 11.85 \\
\hline 5 & 11 & 23.00 & 172.19 \\
\hline
\end{tabular}

Parameters Degree, Closeness and Betweenness are all, to some extent, reflections of the importance of certain vertices in a social network. According to the calculation results of Table 3, students 5 has the largest values of all three parameters in the cohesive subgroup the vertices belongs. Therefore, its importance should not be ignored. Class A Teacher's feedback information confirms that student 5 is indeed one of the core figures in this subgroup. 


\subsection{Class B}

While the class is divided into two major subgroups (Figure 3-(a)), gender is the main determinant factor of division. The subgroups targeted by the research is generally consisted of one. While the class is divided into two major subgroups (Figure 4-(a)), gender is not the determinant factor under this situation.

While the class is divided into three subgroups, a subgroup of distinct characteristics with both genders is formed. Girls number 5, 8, 14 are in this subgroup because, according to the class teacher, they are all outgoing students who associate with boys rather frequently. In the same subgroup with these girls are boys number 6 and 9, who are the core figures of the class with significant leadership ability.

While the class is divided into four subgroups (Figure 4-(b)), the girls in the previous mixed-gender subgroup split from the boys and become a new subgroup, and the boys centered around students 6 and 9 exist independently as well. The situation is mostly similar when there are five cohesive subgroups.

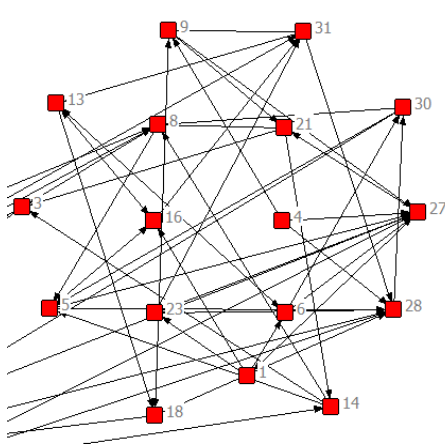

(a)Two Factions

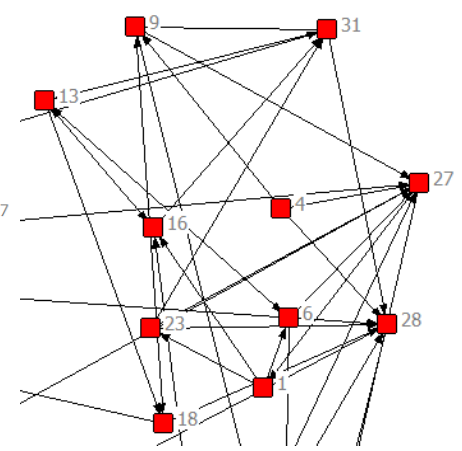

(b) Four Factions

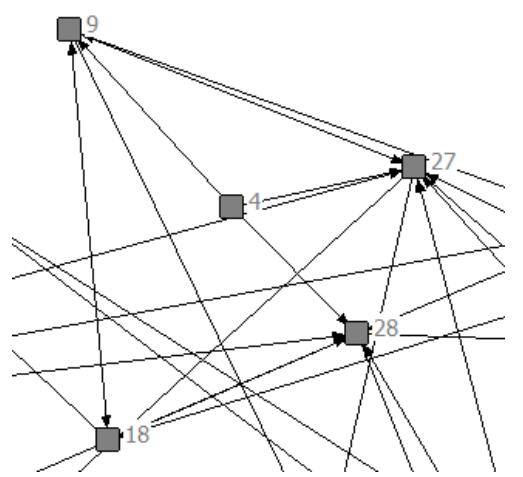

(c) Seven Factions

Figure 4. Division process of cohesive subgroups in class B.

While the class is divided into six or seven even smaller subgroups (Figure 4-(c)), the male subgroup splits into two with student number 6 leading one and number 9 leading another. The members of the subgroup where boy number 6 belongs are all football lovers with medium grades, while the subgroups boy number 9 belongs has student 27 as the core individual.
Calculation of core figures' network parameters by Hubba plug-in in Cytoscape:

Table 3. Hubba calculation of class B core figures' network parameters.

\begin{tabular}{|c|c|c|c|}
\hline Node Name & Degree & Closeness & Betweenness \\
\hline 4 & 3 & 16.25 & 1.16 \\
\hline 18 & 5 & 18.25 & 45.28 \\
\hline 9 & 6 & 18.50 & 18.19 \\
\hline 28 & 9 & 21.67 & 82.39 \\
\hline 27 & 9 & 22.25 & 99.11 \\
\hline
\end{tabular}

Similarly, based on parameters Degree, Closeness and Betweenness that indicate level of importance of vertices in social network, student 27 has the largest values of all three parameters from Table 4, which proves its central status in the corresponding subgroup. The description of this student from Class B Teacher corroborates the discovery.

\subsection{Q-Value Analysis}

While the class is divided into two major subgroups (Figure 3-(a)), gender is the main determinant factor Calculate the q-value of each cohesive subgroup with Girvan-Newman Algorithm. The q-values of both classes are presented in Table 2. The change of q-value with regard to the number of subgroups is presented in Figure 5.

Table 4. Q-values of different numbers of subgroups within both classes.

\begin{tabular}{|l|c|c|c|c|c|c|c|}
\hline & 1 & 2 & 3 & 4 & 5 & 6 & 7 \\
\hline Class A & 0.000 & 0.369 & 0.429 & 0.441 & 0.442 & 0.458 & 0.459 \\
\hline Class B & 0.000 & 0.355 & 0.458 & 0.452 & 0.450 & 0.430 & 0.440 \\
\hline & 8 & 9 & 10 & 11 & 12 & 13 & 14 \\
\hline Class A & 0.453 & 0.447 & 0.432 & 0.423 & 0.405 & 0.386 & 0.372 \\
\hline Class B & 0.431 & 0.424 & 0.381 & 0.365 & 0.339 & 0.333 & 0.325 \\
\hline
\end{tabular}

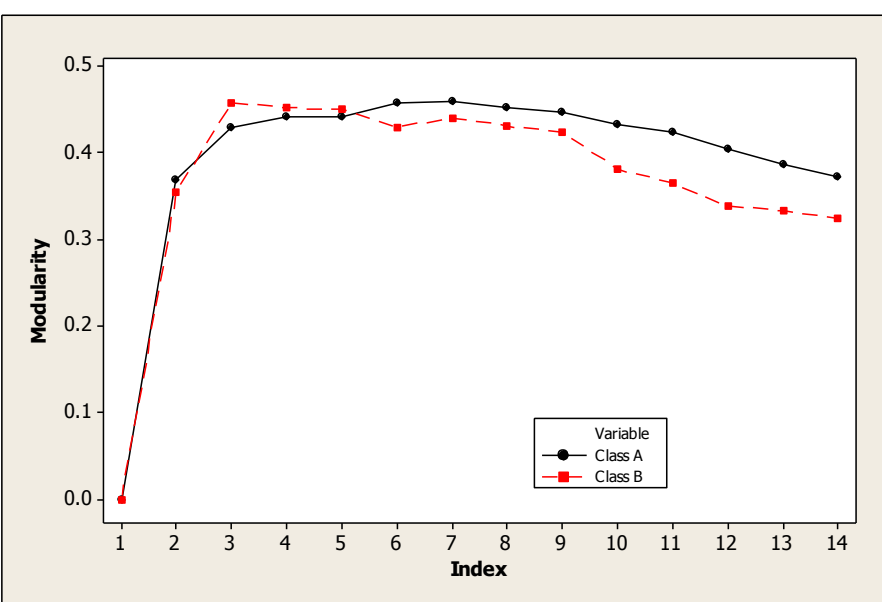

Figure 5. Relationship between q-value and number of cohesive subgroups. 
According to Table 2 and Figure 5, when Class B is divided into 3 subgroups, the q-value is the largest, while q-value for Class A is the largest when it is divided into 7 subgroups.

In conclusion, cohesive subgroups within class are usually organized according to factors including gender, grades, family, personality and hobby. Generally, the relationships among girls tend to be more complicated, so they are likely to divide into more subgroups. Each subgroup is organized around 1-2 core figures and as the size of the subgroup decreases, these strong core figures tend to separate. The new subgroups formed generally have similar complementary relationship inside. The smaller the subgroup, the more powerful the influence of the core figure.

\section{APPLICATION}

The class teachers' overall observation of the relationships among classmates corresponds with most of the analysis results from this study. Understanding of relationships among students from a theoretical perspective is instrumental to the teachers' influence, regulation and management on students. Information distribution mechanism is useful for guidance of public opinion within the class. The concept of cohesive subgroups can be utilized for positive influence from each other in the same faction. Qualities like the most popular and the most unpopular clarify students' social situation and promote effective psychological counseling. These conclusions from social network knowledge will certainly enhance the efficiency of class teachers' work.

Based on the results of experimental analysis, student number 9 in Class A has the smallest degree, which is 3 , and students number $27,28,33,36,40$ and 43 have degree of only 4 . According to theoretical analysis, they are those asocial individuals who do not have the attention of the population (the total number of students who like them and dislike them is the smallest). Teachers should focus on communication with these students, beware of their psychological conditions and encourage them to socialize more actively with their peers. According to the feedback information from Class A teacher, students 9, 28, 33 and 43 are indeed relatively asocial and unconfident. With regard to the same parameter, students 5, 12, 30, 19 and 39 are those with the largest values of degree, and the class teacher confirms that they are relatively popular members of the class. As the supervisor of a class, the teacher can assign to these students tasks like appealing to others or arrange them to sit next to unpopular students and help them involve in socialization with peers. In Class $\mathrm{B}$, with regard to the issue of puppy love, boy number 7 and girl number 12 should be scrutinized because they are most popular among the other gender.

\section{ACKNOWLEDGEMENT}

The authors thank Ms. Yuan Yuan for her significant contribution to the research of this article.

\section{REFERENCES}

[1] References in the text: Figure 1, Figures 2-4, 6, 8a, b (not abbreviated)

[2] Graph Theory and Complex Networks: An Introduction. Maarten van Steen. 5 April 2010.

[3] K. Urberg S. Degirmencioglu, J. Tolson and K. HallidayScher, 1994-2, Psychological Development and Education, Study on Network of Teenage Companion Relationship.

[4] http://www.analytictech.com/ucinet/

[5] http://www.netdraw.com/

[6] http://cytoscapeweb.cytoscape.org/

[7] http://hub.iis.sinica.edu.tw/cytohubba 